\title{
Skin Lesions in a Daclizumab-treated Patient with Multiple Sclerosis
}

\author{
Anca Chiriac ${ }^{1,2,3}$, Adrian Năznean ${ }^{4}$, Cristian Podoleanu ${ }^{5}$, Claudiu Molnar ${ }^{6}$, Simona Stolnicu 7,8 \\ 1 Department of Dermatology, Nicolina Medical Center, Iași, Romania \\ 2 Apollonia University, Iași, Romania \\ 3 "P. Poni” Research Institute, Romanian Academy, Iași, Romania \\ 4 Department of Foreign Languages, University of Medicine, Pharmacy, Science and Technology, Târgu Mureș, Romania \\ 5 Department of Internal Medicine, University of Medicine, Pharmacy, Science and Technology, Târgu Mureș, Romania \\ 6 Department of Gynecology, University of Medicine, Pharmacy, Science and Technology, Târgu Mureș, Romania \\ 7 Department of Pathology, University of Medicine, Pharmacy, Science and Technology, Târgu Mureș, Romania \\ 8 Histopat Invest Laboratory, Târgu Mureș, Romania
}

\section{CORRESPONDENCE}

\section{Cristian Podoleanu}

Str. Gheorghe Marinescu nr. 38

540139 Târgu Mureș, Romania

Tel: +40 265215551

E-mail: podoleanu@me.com

\section{ARTICLE HISTORY}

Received: September 1, 2018

Accepted: December 12, 2018
Anca Chiriac • Str. Hatman Șendrea nr. 2, 700613 lași, Romania. Tel: +40332808703

Adrian Năznean • Str. Gheorghe Marinescu nr. 38 540139 Târgu Mureș, Romania. Tel: +40 0265215551 Claudiu Molnar • Str. Gheorghe Marinescu nr. 38 , 540139 Târgu Mureș, Romania. Tel: +40 0265215551 Simona Stolnicu • Str. Gheorghe Marinescu nr. 38 540139 Târgu Mureș, Romania. Tel: +40 0265215551

\begin{abstract}
Background: Daclizumab is a humanized monoclonal antibody against the $\alpha$-subunit (CD25) of the high-affinity interleukin (IL)-2 receptor, used for the treatment of relapsing-remitting multiple sclerosis with a large spectrum of cutaneous adverse reactions. Case presentation: We present the case of a middle-aged man treated with daclizumab for multiple sclerosis, who developed skin reactions difficult to evaluate. A $4 \mathrm{~mm}$ punch-biopsy was taken from the plantar area. Histological examination of the biopsy revealed hyperkeratosis and acanthosis but no parakeratosis, while a discrete inflammatory infiltrate was noticed around vessels in the dermis. Treatment with fluconazole $50 \mathrm{mg} /$ day for 10 days, moisturizers, and grade I topical steroids was followed by slight improvement of the clinical picture. Treatment with daclizumab was not discontinued. Conclusion: The clinical efficacy and side effects of daclizumab have to be reported and confirmed in clinical practice in the following years. Any clinical report can contribute to validate the efficacy and risk of the drug's administration. Any type of adverse skin reaction must be reported for clarifying the diagnosis.
\end{abstract}

Keywords: daclizumab, skin lesions, multiple sclerosis

\section{BACKGROUND}

Daclizumab is a humanized monoclonal antibody against the $a$-subunit (CD25) of the high-affinity interleukin (IL)-2 receptor, used for the treatment of relapsing-remitting multiple sclerosis, with a large spectrum of cutaneous adverse reactions. ${ }^{1,2}$ Physicians should be aware of potential side effects induced by daclizumab in the treatment of patients with relapsing multiple sclerosis. We present the case of a middle-aged man treated with daclizumab for multiple sclerosis, who developed skin reactions difficult to evaluate. 


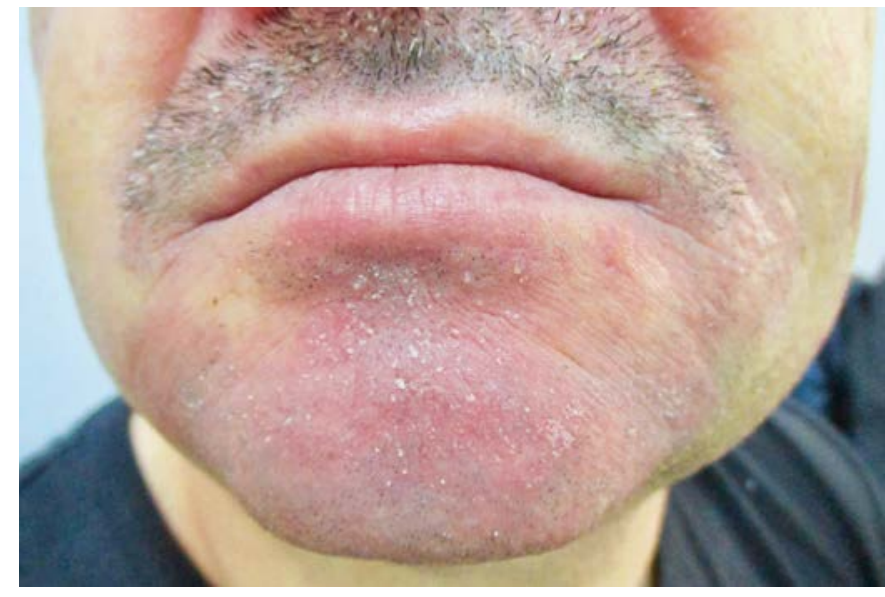

FIGURE 1. Large erythematous plaques covered by scales over the chin and on the middle area of the face; inferior blepharitis

\section{CASE PRESENTATION}

A 56-year-old man, treated with daclizumab for multiple sclerosis, was referred to Dermatology. The dermatological examination revealed an intensely pruritic desquamating rash localized on the central facial area (mostly on the chin and nasal wings) associated with inferior blepharitis (Figure 1); androgenic alopecia and discrete silver scales over the parieto-temporal area (Figure 2); nail pitting, discrete distal onycholysis and chronic perionixis on all digits (Figure 3); bilateral plantar hyperkeratosis (Figure 4); no other skin lesions were observed or described by the patient. He admitted having the alopecia from the second decade of life, as well as a long history of cutane-

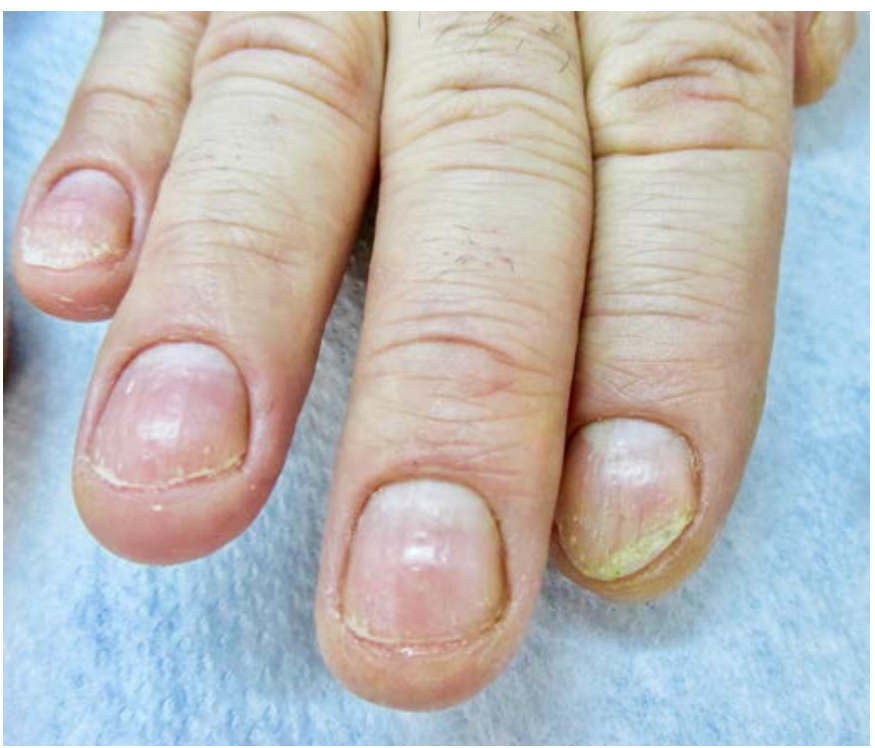

FIGURE 3. Nail pitting, discrete distal onycholysis and chronic perionixis on all digits

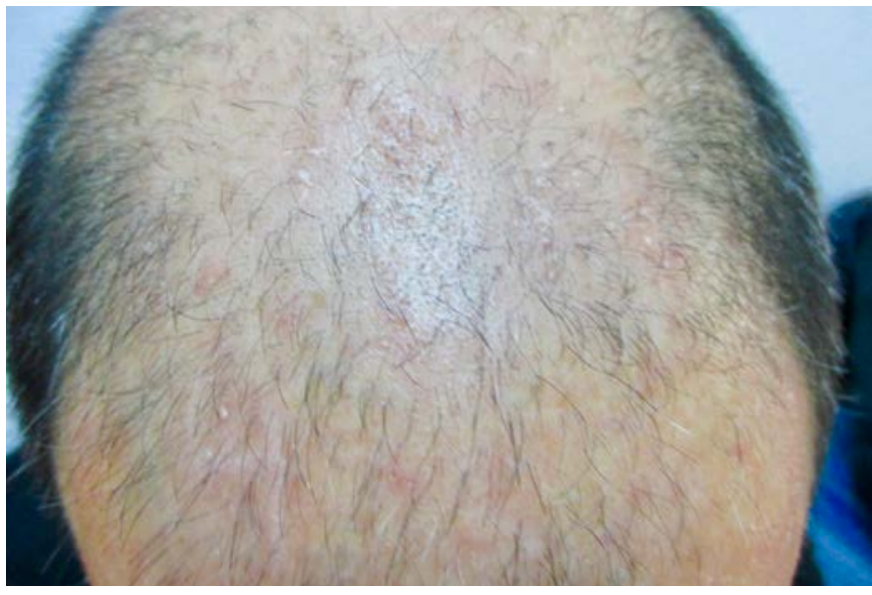

FIGURE 2. Androgenic alopecia and discrete silver scales over the parieto-temporal area

ous problems, and a recent facial rash forced him to seek treatment.

Before referral to the hospital, emollients were used regularly as self-medication for the last 2 months, with no improvement. No personal or familial medical history of psoriasis was declared or recorded in medical files.

A clinical suspicion of seborrheic dermatitis or psoriasis motivated a skin biopsy. A $4 \mathrm{~mm}$ punch-biopsy was taken from the plantar area. Histological examination of the biopsy revealed hyperkeratosis and acanthosis but no parakeratosis, while a discrete inflammatory infiltrate was noticed around vessels in the dermis. Treatment with fluconazole $50 \mathrm{mg}$ /day for 10 days, moisturizers, and grade I topical steroids was followed by slight improvement of the clinical manifestations. Treatment with daclizumab was not discontinued.

A month later, the patient was seen again; typical small patches of seborrheic-like dermatitis were observed on the sternal area (Figure 5) and on the scapular area (Figure 6), while the old cutaneous lesions were still pres-

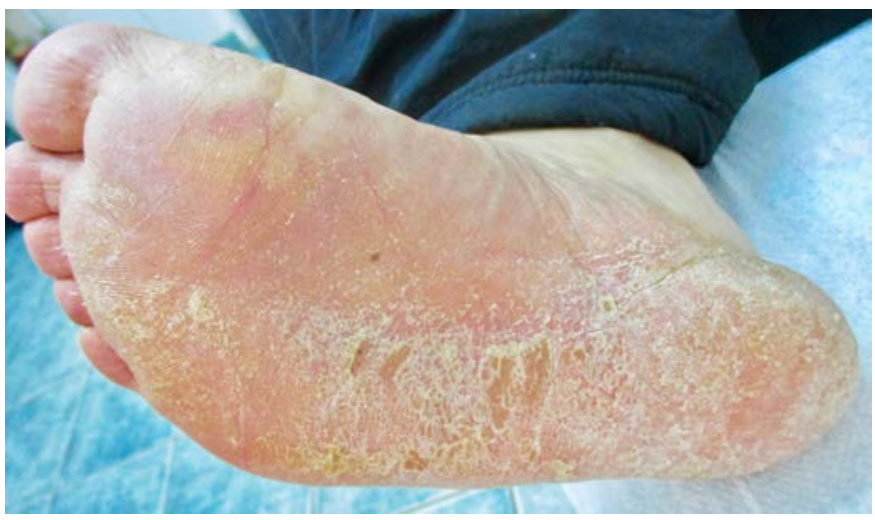

FIGURE 4. Hyperkeratosis covering the entire plantar surface 


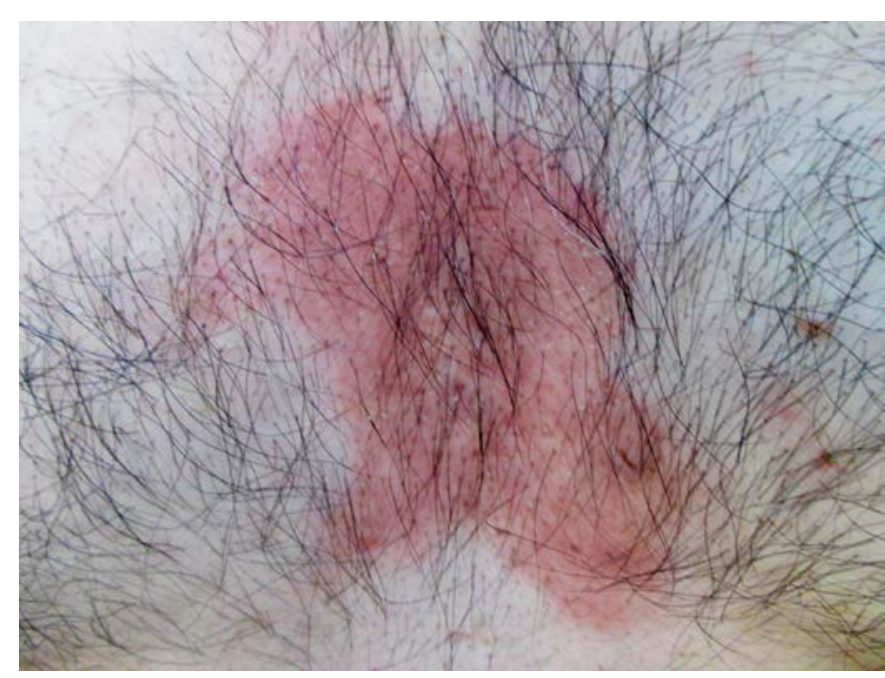

FIGURE 5. Seborrheic-like lesions on the sternal area

ent. Potent topical steroids were recommended for two weeks for the scalp and trunk lesions, associated with $10 \%$ urea cream for the plantar area, ketoconazole gel as cleaning agent for the face, followed by emollients. No restriction for daclizumab treatment was recommended from a dermatological point of view, taking into consideration the lack of severity of skin lesions and the beneficial effect of daclizumab on his neurological disease. The patient will be closely followed-up with an interdisciplinary approach.

\section{DISCUSSIONS}

Daclizumab is a humanized monoclonal antibody against the $a$-subunit (CD25) of the high-affinity interleukin (IL)2 receptor used for the treatment of relapsing-remitting multiple sclerosis. ${ }^{1}$ Recently, several reports have communicated a large spectrum of cutaneous adverse reactions observed in patients treated with daclizumab for multiple sclerosis. ${ }^{2}$

Cutaneous adverse reactions induced by daclizumab high-yield process (DAC-HYP), recommended for multiple sclerosis, have been previously reported in $37 \%$ of patients, mainly represented by "rash and eczema". ${ }^{2}$ Another open-label phase I study of DAC-HYP reported that $77 \%$ of the enrolled patients presented skin adverse reactions, described clinically as "severe rash of psoriasiform phenotype" but with no characteristic histological features. ${ }^{3}$ The severity of skin reactions was mild to moderate, with good response to topical steroids, and rare cases of severe reactions, such as angioedema or Stevens-Johnson syndrome, were reported. ${ }^{4,5}$ In a recent study performed on 2,236 patients with 5,214 patient-years of exposure to daclizumab

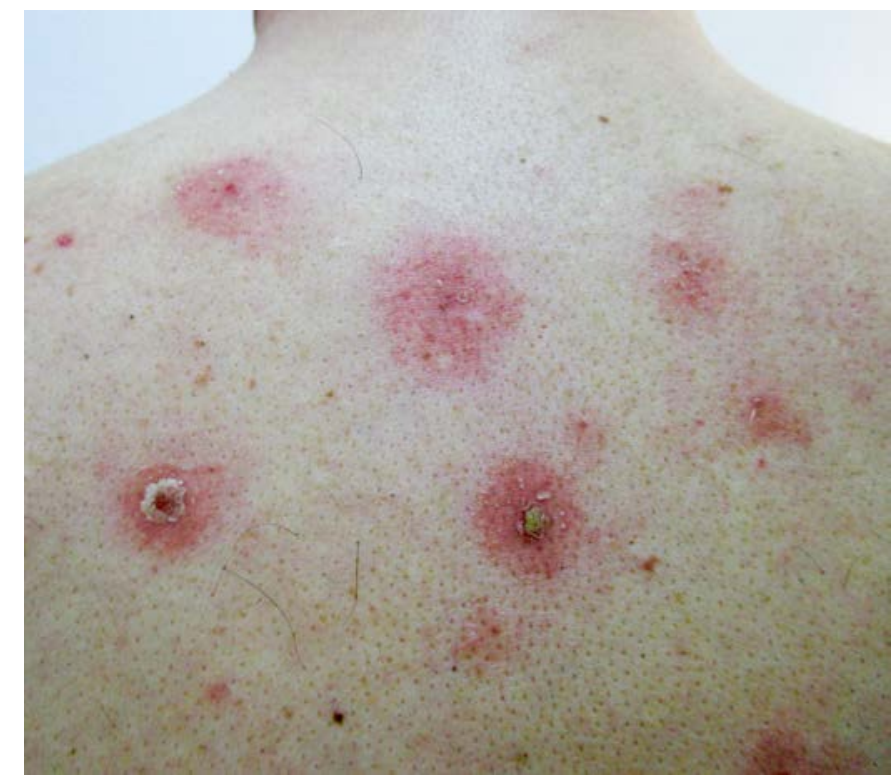

FIGURE 6. Seborrheic-like lesions on the scapular area

for relapsing-remitting multiple sclerosis, the cumulative incidence of skin adverse reactions was found in $33 \%$ of patients, represented by rash, eczema, and allergic dermatitis, with rare (only $2 \%$ ) cases of severe adverse events (toxic skin eruption treated with systemic steroids and plasma exchange). ${ }^{5}$

Daclizumab treatment in multiple sclerosis expands CD56bright NK cells; this same lymphocyte subset is involved in the pathogenesis of psoriasis and may lead to psoriasis in genetically predisposed patients. ${ }^{6,7}$

Our patient had developed inflammatory skin lesions during treatment with daclizumab, but the exact diagnosis was not ascertained. Although clinical features argued in favor of psoriasis, histology did not certify the diagnosis. Moreover, in some cases, a clear distinction between psoriasis and seborrheic dermatitis cannot be made, or both diseases can coexist in the same patient. Daclizumab was approved for use outside of clinical studies in May 2016; its clinical efficacy and side effects have to be reported and confirmed in clinical practice in the following years. ${ }^{7}$

\section{CONCLUSIONS}

Psoriasis-like skin lesions may present as adverse effects of treatment with Daclizumab in patients with relapsingremitting multiple sclerosis. Any clinical report can contribute to validate the efficacy and risk of the drug's administration. Any type of adverse skin reaction must be reported for clarifying the diagnosis. 


\section{CONSENT TO PARTICIPATE}

Informed consent to publish the case was obtained from the patient.

\section{CONFLICT OF INTEREST}

None for all authors.

\section{REFERENCES}

1. Bielekova B. Daclizumab therapy for multiple sclerosis. Neurotherapeutics. 2013;10:55-67.
2. Krueger JG, Kircik L, Hougeir F, et al. Cutaneous Adverse Events in the Randomized, Double-Blind, Active-Comparator DECIDE Study of Daclizumab High-Yield Process Versus Intramuscular Interferon Beta-1a in Relapsing-Remitting Multiple Sclerosis. Adv Ther. 2016;33:1231-1245

3. Cortese I, Ohayon J, Fenton $\mathrm{K}$, et al. Cutaneous adverse events in multiple sclerosis patients treated with daclizumab. Neurology. 2016;86:847-855.

4. Kircik L, Krueger J, Lebwohl M, et al. Incidence, severity, duration, and treatment of cutaneous adverse events in the DECIDE study of daclizumab HYP versus intramuscular interferon beta-1a in patients with relapsingremitting multiple sclerosis. Mult Scler. 2015:21:251-252.

5. Gold R, Giovannoni G, Selmaj K, et al. Daclizumab high-yield process in relapsing-remitting multiple sclerosis (SELECT): a randomised, doubleblind, placebo-controlled trial. Lancet. 2013;381:2167-2175.

6. Diao L, Hang Y, Othman AA, et al. Population PK-PD analyses of CD25 occupancy, CD56bright NK cell expansion, and regulatory T cell reduction by daclizumab HYP in subjects with multiple sclerosis. Br J Clin Pharmacol. 2016;82:1333-1342

7. Michel T, Poli A, Cuapio A et al. Human CD56bright NK Cells: An Update. J Immunol. 2016; 196:2923-2931. 